\title{
The cross sections and energy spectra of the particle emission in proton induced reaction on ${ }^{204,206,207,208} \mathrm{~Pb}$ and ${ }^{209} \mathrm{Bi}$
}

\author{
Zhengjun Zhang ${ }^{1}$, Haiying Liang ${ }^{2}$, Yongli Xu ${ }^{3}$, Yinlu $\mathrm{Han}^{2, \mathrm{a}}$, Chonghai Cai ${ }^{4}$, and Qingbiao Shen ${ }^{2}$ \\ 1 Department of Physics, Northwest University, Xi'an 710069, P.R. China \\ 2 Key Laboratory of Nuclear Data, China Institute of Atomic Energy, PO Box 275(41), Beijing 102413, P.R. China \\ 3 School of Physics and Electronic Science, Shanxi Datong University, Datong 037009, P.R. China \\ ${ }^{4}$ Department of Physics, Nankai University, Tianjin 300071, P.R. China
}

\begin{abstract}
All cross sections of proton induced reactions, angular distributions, energy spectra and double differential cross sections of neutron, proton, deuteron, triton, helium and alpha-particle emissions for $\mathrm{p}+{ }^{204,206,207,208} \mathrm{~Pb},{ }^{209} \mathrm{Bi}$ reactions are consistently calculated and analyzed at incident proton energies below $200 \mathrm{MeV}$. The optical model, the distorted wave Born approximation theory, the unified Hauser-Feshbach and exciton model which includes the improved Iwamoto-Harada model are used. Theoretically calculated results are compared with the existing experimental data.
\end{abstract}

\section{Introduction}

New accelerator-driven technology that utilizes spallations, such as the production of tritium and the transmutation of radioactive waste, is a growing interest in such type of reactions especially due to the emerging new ideas concerning the hybrid systems. Such systems are supposed to use intense high-energy proton beams, which induce spallation reactions on heavy targets. A large number of high-energy neutrons along with light charged particles are produced. The Accelerator-Driven System (ADS) requires nuclear data of common cross sections and especially the data of neutron and proton induced energy-angle correlated spectra of secondary light particles as well as double differential cross sections to model the performance of the target/blanket assembly and to predict neutron production, activation, heating, shielding requirements, and material damage. Due to specific experimental difficulties, experimental results concerning light charged particle production in proton induced reactions are rather scarce. The development of high-quality nuclear data for Lead and Bismuth is particularly important due to their role as an important target material in many accelerator-driven system designs.

In this work, all cross sections, angular distributions, and angle-integrated spectra of particles emission for $\mathrm{p}+{ }^{204,206,207,208} \mathrm{~Pb},{ }^{209} \mathrm{Bi}$ reactions are consistently calculated by using the optical model [1], the distorted wave Born approximation theory [2], the unified Hauser-Feshbach and exciton model [3]. The improved Iwamoto-Harada model [4-7] is included in the exciton model to provide a comprehensive and improved description of the energy spectra and double differential cross sections of the light composite particles $\left(\mathrm{d}, \mathrm{t},{ }^{3} \mathrm{He}\right.$ and $\alpha$ ) emission at incident proton energy up to $200 \mathrm{MeV}$.

a e-mail: hanyl@ciae.ac.cn
Section 2 provides a description of the theoretical models used in this work. Section 3 gives the analysis and comparisons of the calculated results with the experimental data. Sect. 4 gives a simple conclusion.

\section{Theoretical models and parameters}

The optical model is used to describe the measured proton-induced total reaction cross sections and elastic scattering angular distributions, and to calculate the transmission coefficient of the compound nucleus and the pre-equilibrium emission process for all particles (neutron, proton, deuteron, triton, helium and alphaparticle). The optical model potentials considered here are Woods-Saxon [1] type for the real part, WoodsSaxon and derivative Woods-Saxon type for the imaginary parts corresponding to the volume and surface absorptions and the Thomas form for the spin-orbit part, respectively.

The theoretical model code APMN [8] is used to obtain the optical model potential parameters. The optical potential parameters for proton are obtained from the experimental data of total reaction cross section and elastic scattering angular distributions for $\mathrm{p}+{ }^{208} \mathrm{~Pb}$ and ${ }^{209} \mathrm{Bi}$ reactions at incident proton energy up to $250 \mathrm{MeV}$. A set of neutron optical model potential parameters [9] for $\mathrm{n}+{ }^{208} \mathrm{~Pb}$ and ${ }^{209} \mathrm{Bi}$ reactions are used in incident neutron energy from 0.1 to $250 \mathrm{MeV}$. The optical potential parameters for deuteron, triton, helium and alpha-particle are taken from the global optical potential parameters [10-13] with incident energies from threshold up to $200 \mathrm{MeV}$, based on the experimental data of particle total reaction cross sections and elastic scattering angular distributions, respectively.

The direct inelastic scattering angular distributions to low-lying states are important in nuclear data theoretical calculations. The code DWUCK4 [2] of the distorted wave Born approximation theory is used to pre-calculate 


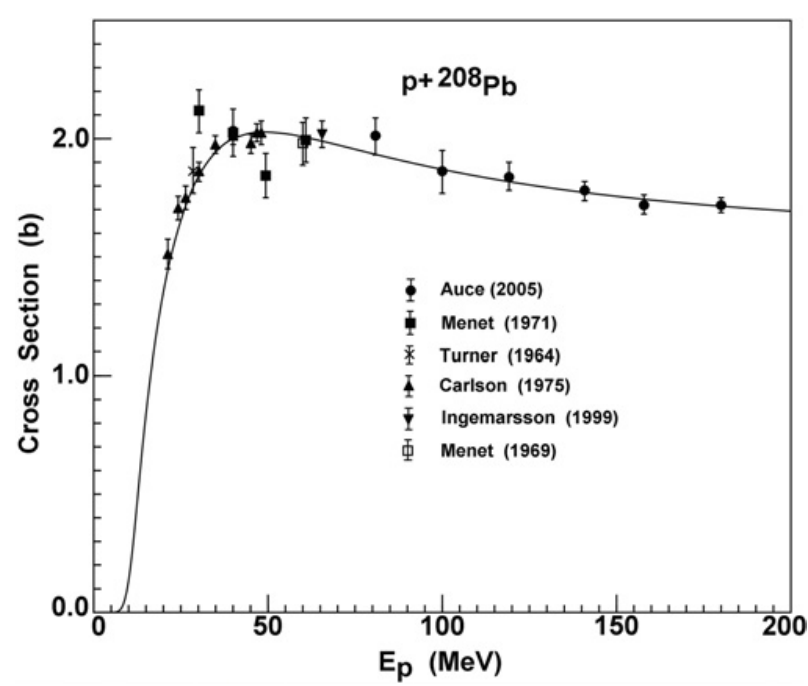

Figure 1. Calculated reaction cross sections compared with the experimental data of $\mathrm{p}+{ }^{208} \mathrm{~Pb}$ reaction.

the direct inelastic scattering cross sections and angular distributions of discrete levels. The experimental data of inelastic scattering angular distributions for discrete levels are used to guide theoretical calculations. The deformation parameters for excited levels are obtained.

The unified Hauser-Feshbach and exciton model are used to describe the nuclear reaction equilibrium and preequilibrium decay processes. The Hauser-Feshbach model with width fluctuation correction is used to describe the emissions from compound nucleus to the discrete levels and continuum states of the residual nuclei in equilibrium processes, and the preequilibrium process is described by the angular momentum dependent exciton model. The emissions to the discrete level and continuum states in the multi-particle emissions for all opened channels are included. The preequilibrium statistical theory based on the exciton model, evaporation models and the HauserFeshbach theory with width fluctuation correction, and the intranuclear cascade model are used to describe the nuclear reaction preequilibrium and equilibrium decay processes. The improved Iwamoto-Harada model is used to describe the composite particle $\left(\mathrm{d}, \mathrm{t},{ }^{3} \mathrm{He}\right.$ and $\alpha$ ) emission in compound nucleus.

The nuclear reaction models code MEND [14], which can give all kinds of reaction cross sections and energy spectra for six outgoing light particles (neutron, proton, alpha, deuteron, triton, and helium) and various residual nuclei in the energy range up to $250 \mathrm{MeV}$, is used.

The level density parameters and pair correction parameters of the Back-Shifted Fermi gas level density [15] for low energy are used. The Ignatyuk nuclear level densities [16] are used, which include the washing-out of shell effects with increasing excitation energy, collective excitations as well as single particle ones, departs from more traditional ones, and are matched continuously onto low-lying experimental discrete level. The Ignatyuk model for describing the statistical level density properties of excited nuclei is particularly appropriate for the relatively high energies. The parameters of the level densities and pair correction are taken from RIPL [17], and adjusted by fitting the experimental data of some channel reaction cross sections.

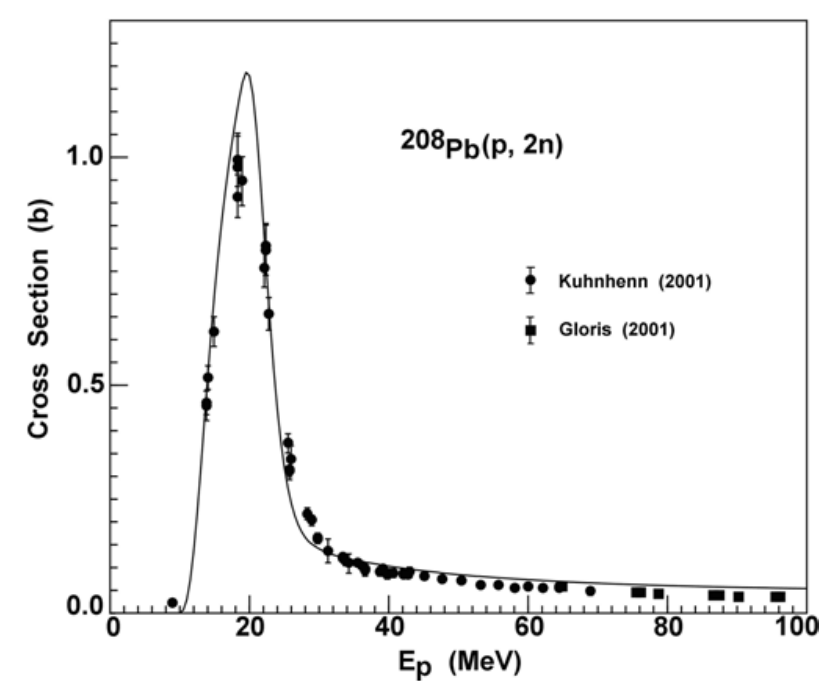

Figure 2. Calculated ${ }^{208} \mathrm{~Pb}(\mathrm{p}, 2 \mathrm{n}){ }^{207} \mathrm{Bi}$ reaction cross sections compared with the experimental data.

\section{Theoretical results and analysis}

The theoretical calculated results of total reaction cross sections for $\mathrm{p}+{ }^{208} \mathrm{~Pb}$ are in good agreement with the experimental data as shown in Fig. 1. There are no experimental data of proton total reaction cross sections for $\mathrm{p}+{ }^{209} \mathrm{Bi}$ reaction. The theoretical calculated results also agree with the experimental data of $\mathrm{p}+{ }^{208} \mathrm{~Pb}$ reaction. The comparison of the calculated results of elastic scattering angular distributions with the experimental data for $\mathrm{p}+{ }^{204,206,208} \mathrm{~Pb},{ }^{209} \mathrm{Bi}$ reactions shows that the calculated results consist with the experimental data. The calculated results of proton inelastic scattering angular distributions for different discrete levels of different targets at different incident energy are compared with the experimental data. The calculated results are in good accordance with the experimental data.

The comparison of the calculated results of proton induced reaction cross section for different channels of different targets with the experimental data shown that the calculated results are in good or reasonable agreement with the experimental data. The comparison of some calculated results with the experimental data are only given in Figs. 2 to 4 . The disagreements between the calculated results and the experimental data for some reaction channels need to be studied theoretically and experimentally in the future.

Based on the agreements of the calculated results with the experimental data above all reaction cross sections and angular distributions, the energy spectrum of neutron, proton, deuteron, triton, helium and alpha emission for $\mathrm{p}+{ }^{204,206,207,208} \mathrm{~Pb},{ }^{209} \mathrm{Bi}$ reactions are calculated by theoretical models.

The calculated results of the energy spectra of neutron emission for $\mathrm{p}+{ }^{204,206,207,208} \mathrm{~Pb}$ reactions are compared with the experimental data [18] at incident proton energy $25.5 \mathrm{MeV}$ as shown in Fig. 5. The calculated results of the energy spectra of neutron, proton, deuteron, triton helium and alpha emission for $\mathrm{p}+{ }^{208} \mathrm{~Pb}$ reaction are compared with the experimental data [19] at incident proton energy $62.9 \mathrm{MeV}$ in Fig. 6. The magnitude and shape of theoretical calculated curve of energy spectra for neutron, proton, deuteron, triton and helium emission is in accordance with the experimental data. 


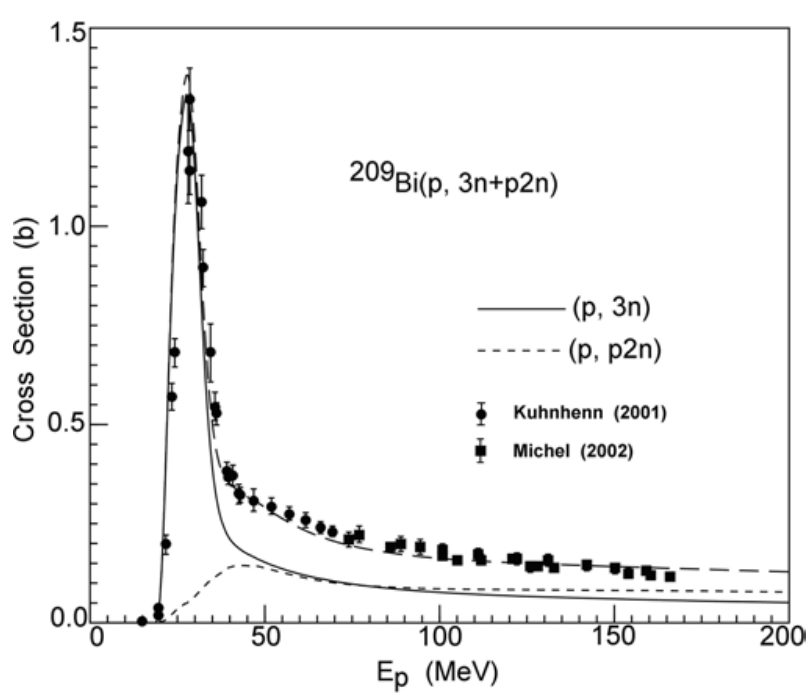

Figure 3. Calculated ${ }^{209} \mathrm{Bi}(\mathrm{p}, 3 \mathrm{n}+\mathrm{p} 2 \mathrm{n})$ reaction cross sections compared with the experimental data.

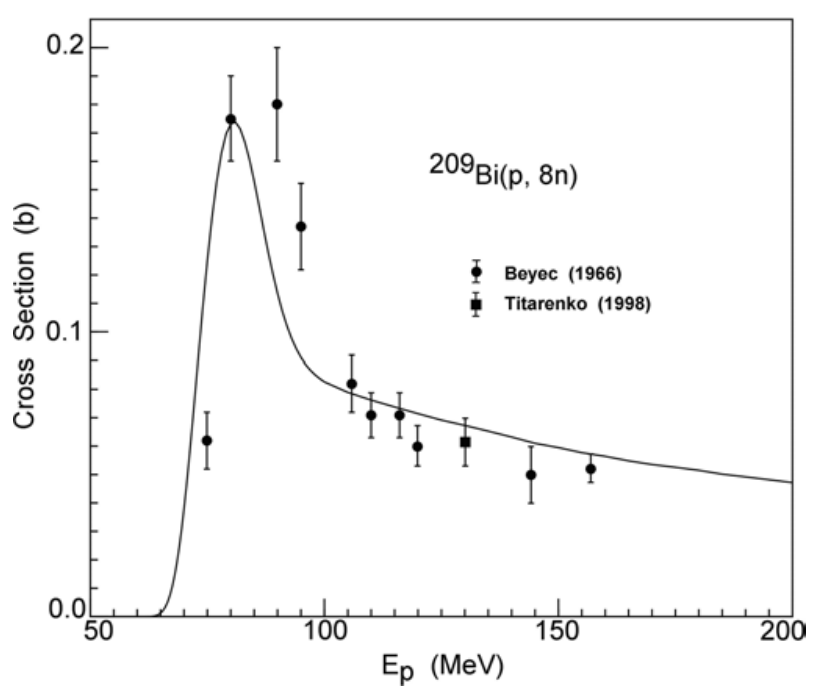

Figure 4. Calculated ${ }^{209} \mathrm{Bi}(\mathrm{p}, 8 \mathrm{n}){ }^{202}$ Po reaction cross sections compared with the experimental data.

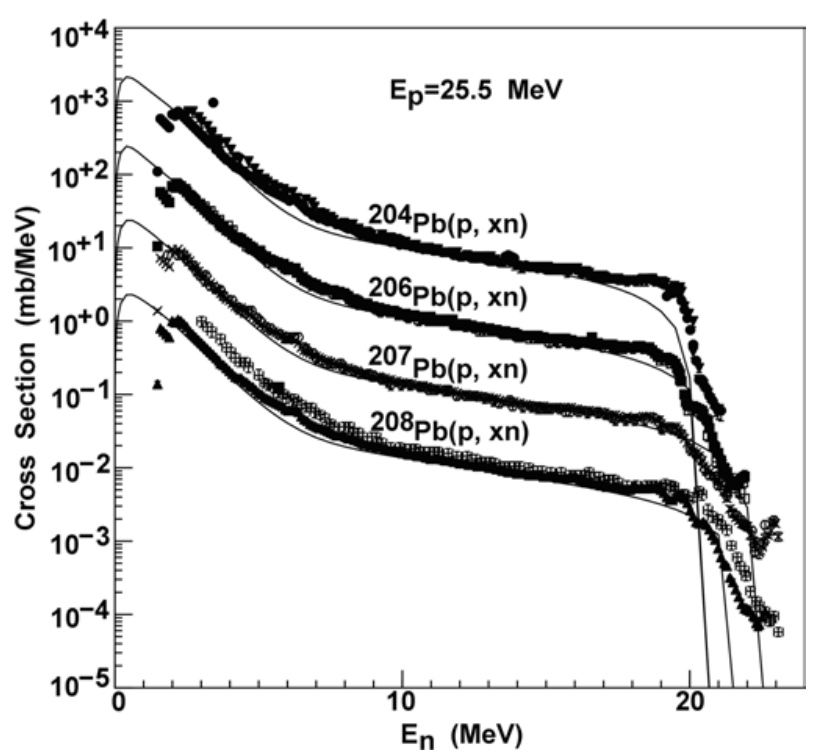

Figure 5. Calculated neutron energy spectra compared with the experimental data for $\mathrm{p}+{ }^{204,206,207,208} \mathrm{~Pb}$ reactions.

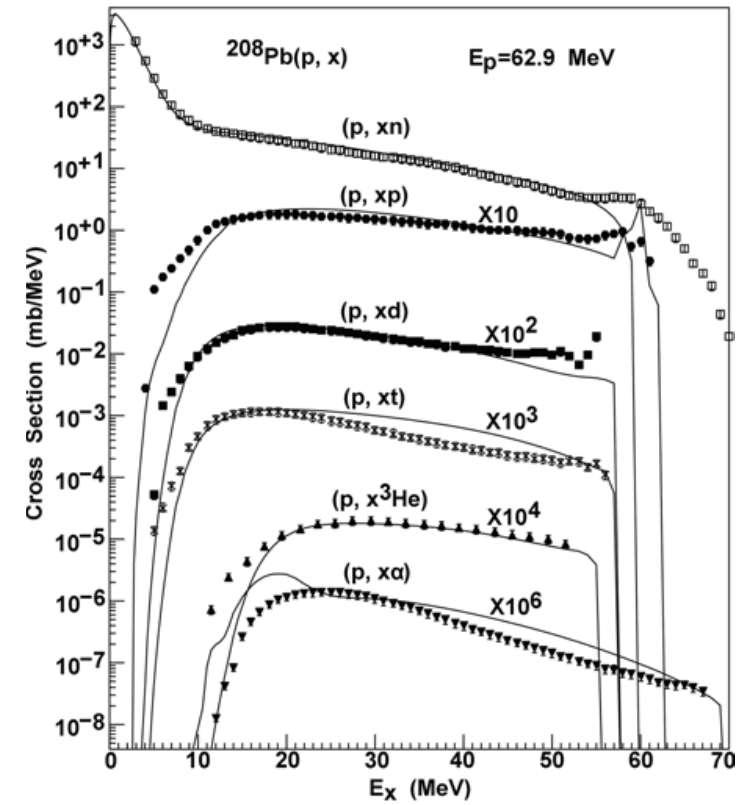

Figure 6. Calculated energy spectra compared with the experimental data for $\mathrm{p}+{ }^{208} \mathrm{~Pb}$ reaction.

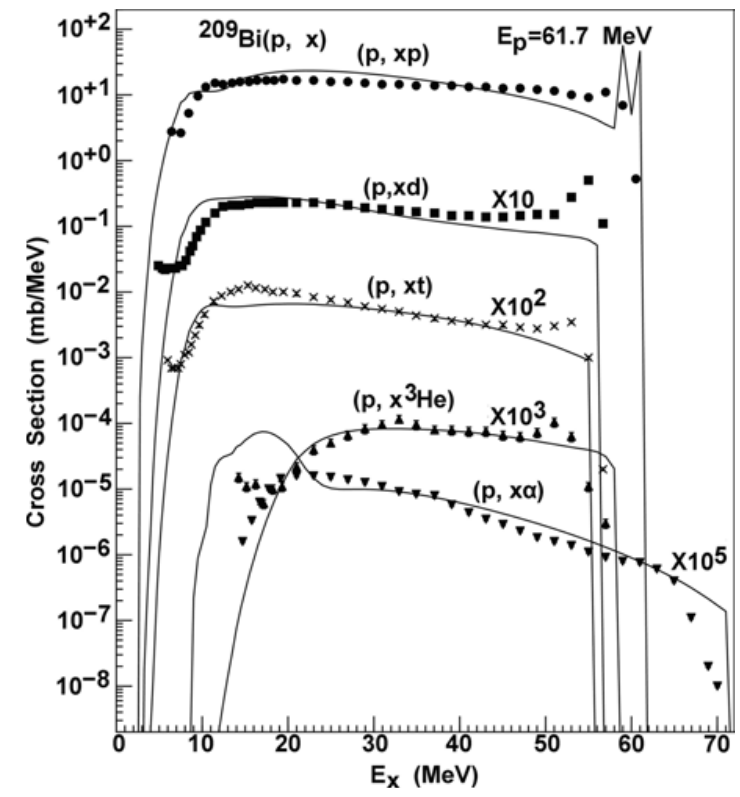

Figure 7. Calculated energy spectra compared with the experimental data for $\mathrm{p}+{ }^{209} \mathrm{Bi}$ reaction.

The theoretical calculated results for alpha emission are in agreement with the experimental data above alpha emission energy $25.0 \mathrm{MeV}$, where are the contributions of the preequilibrium reactions in theoretical calculated results.

The calculated results of the energy spectra of proton, alpha, deuteron, triton and helium emission for $\mathrm{p}+{ }^{209} \mathrm{Bi}$ reaction are compared with the experimental data [20] at incident proton energy $61.7 \mathrm{MeV}$ in Fig. 7. The magnitude and shape of theoretical calculated curve of energy spectra for proton, deuteron, triton and helium emissions are in agreement with the experimental data. The theoretical calculated results for alpha emission agree with the experimental data above alpha emission energy $25.0 \mathrm{MeV}$, and are larger than the experimental data below alpha 


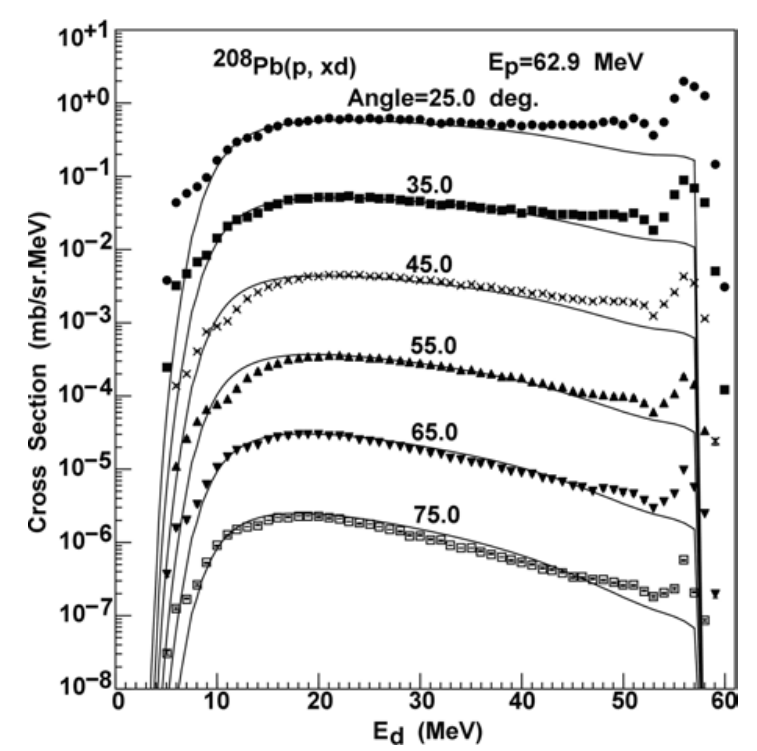

Figure 8. Calculated double differential cross sections of deuteron emission compared with the experimental data for $\mathrm{p}+{ }^{208} \mathrm{~Pb}$ reaction.

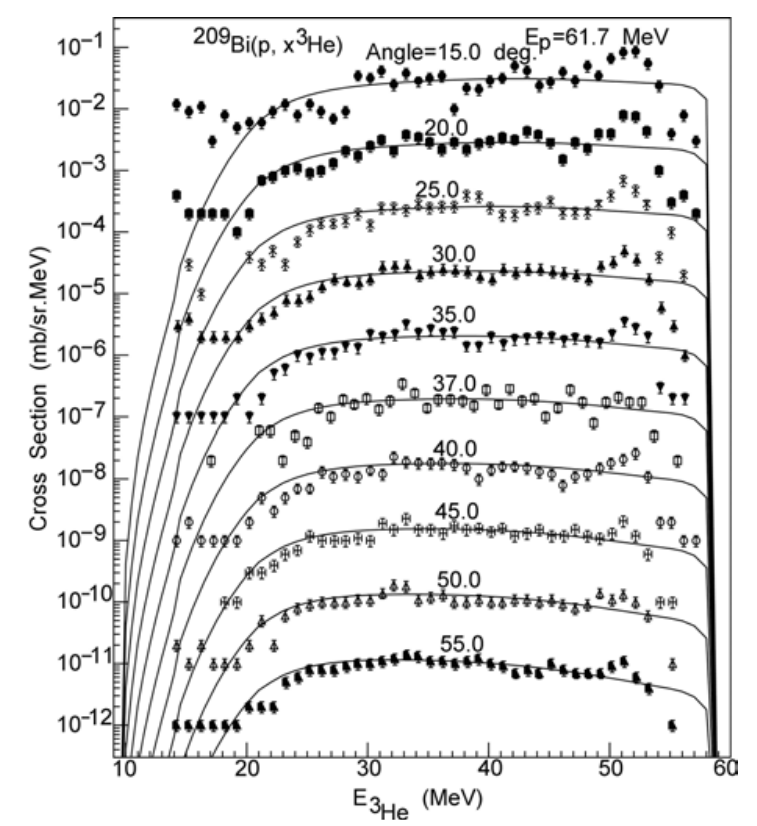

Figure 9. Calculated double differential cross sections of helium emission compared with the experimental data for $\mathrm{p}+{ }^{209} \mathrm{Bi}$ reaction.

emission energy $25.0 \mathrm{MeV}$, where are the contributions of the equilibrium reaction in theoretical calculated results.

The calculated results of the double differential cross sections for deuteron emission for $\mathrm{p}+{ }^{208} \mathrm{~Pb}$ reaction are compared with the experimental data at incident proton energy $62.9 \mathrm{MeV}$ in Fig. 8. The magnitude and shape of the calculated curve are in good agreement with the experimental data [19].
The calculated results of the double differential cross sections for helium emission are compared with the experimental data at incident proton energy $61.7 \mathrm{MeV}$ as shown in Fig. 9. The magnitude and shape of the calculated results curve are in good agreement with the experimental data [20].

\section{Conclusions}

All cross sections of proton induced reaction, angular distributions, energy spectra and double differential cross sections are consistently calculated by using nuclear theory models for $\mathrm{p}+{ }^{204,206,207,208} \mathrm{~Pb},{ }^{209} \mathrm{Bi}$ reactions at incident proton energies from threshold energy to $200 \mathrm{MeV}$. Good agreements are generally observed between the calculated results and the experimental data. Since the improved Iwamoto-Harada model has been included in the exciton model for the light composite particle emissions, the theoretical models provide a good description of the shapes and magnitude of the energy spectra and double differential cross section of emission deuteron, triton, helium and alpha. These data can be effectively used in different practical applications.

This work was part of National Basic Research Program of China (973 Program), and was supported by the China Ministry of Science and Technology under Contract No. 2007CB209903.

\section{References}

[1] JR. F.D. Becchetti, G.W. Greenlees, Phys. Rev. 182, 1190 (1969)

[2] P.D. Kunz, Distorted Wave Code DWUCK4. University of Colorado. 1994

[3] Jingshang Zhang, Nucl. Sci. Eng. 114, 55 (1993)

[4] A. Iwamoto, K. Harada, Phys. Rev. C 26, 1821 (1982)

[5] J.S. Zhang, et al., Z. Phys. A 344, 251 (1992)

[6] J.S. Zhang, Nucl. Sci. Eng. 116, 35 (1994)

[7] Qingbiao Shen, Nucl. Sci. Eng. 117, 99 (1994)

[8] Qingbiao Shen, Nucl. Sci. Eng. 141, 78 (2002)

[9] Yinlu Han, et al., Nucl. Sci. Eng. 150, 78 (2004)

[10] Yinlu Han, et al., Phys. Rev. C 74, 044615 (2006)

[11] Yongli Xu, Hairui Guo, Yinlu Han, Qingbiao Shen, Sci. China Phys. Mech. Astron. 54, 2005 (2011)

[12] Yongli Xu, Hairui Guo, Yinlu Han, Qingbiao Shen, Int. J. Mod. Phys. E 24, 1550005 (2015)

[13] Xinwu Su, Yinlu Han, Int. J. Mod. Phys. E 24, 1550092 (2015)

[14] Chong-hai Cai, Nucl. Sci. Eng. 153, 93 (2006)

[15] W. Dilg, et al., Nucl. Phys. A 217, 269 (1973)

[16] A.V. Ignatyuk, et al., Sov. J. Nucl. Phys. 21, 255 (1975)

[17] R. Capote, et al., Nucl. Data Sheets 110, 3107 (2009)

[18] K. Harder, et al., Phys. Rev. C 36, 834 (1987)

[19] A. Guertin, et al., Eur. Phys. J. A 23, 49(2005)

[20] F.E. Bertrand, R.W.Peelle, Phys. Rev. C 8, 1045 (1973) 